\title{
Transient response of multilayered hollow cylinder using various theories of generalized thermoelasticity
}

\author{
Daoud S. Mashat ${ }^{1}$, Aahraf M. Zenkour ${ }^{1,2 *}$, Khaled A. Elsibai ${ }^{3}$ \\ ${ }^{1}$ Department of Mathematics, Faculty of Science, King AbdulAziz University, Jeddah, Saudi Arabia; ${ }^{*}$ Corresponding Author: \\ zenkour@kau.edu.sa; \\ ${ }^{2}$ Department of Mathematics, Faculty of Science, Kafrelsheikh University, Kafr El-Sheikh, Egypt; \\ ${ }^{3}$ Department of Mathematics, Faculty of Applied Science, Umm Al-Qura University, Holy Makkah, Saudi Arabia.
}

Received 7 March 2010; revised 12 April 2010; accepted 16 April 2010.

\begin{abstract}
The present paper deals with thermoelastic problems of finitely long hollow cylinder composed of two different materials with axial symmetry. The medium is traction-free, with negligible body forces and with internal and external heat generations. The governing equations for different theories of the generalized thermoelasticity are written in terms of displacement and temperature increment. The exact solution of the problem, using different theories of generalized thermoelasticity, has been deduced. The analytical expressions for displacements, temperature and stresses are found in final forms, and a numerical example has been taken to discuss the effect of the relaxation times. Finally, the results have been illustrated graphically to find the responses of different theories.
\end{abstract}

Keywords: Multilayered Hollow Cylinder; Generalized Theories of Thermoelasticity; Relaxation Times

\section{INTRODUCTION}

The governing equations for displacement and temperature fields in the linear dynamical theory of classical thermoelasticity consist of the coupled partial differential equation of motion and Fourier's law of heat conduction equation. The equation for displacement field is controlled by a wave type hyperbolic equation, whereas that for the temperature field is a parabolic diffusion type equation. This amounts to the remark that the classical thermoelasticity predicts a finite speed for predominantly elastic disturbances but an infinite speed for predominantly thermal disturbances, which are coupled together. This means that a part of every solution of the equations extends to infinity.
Biot [1] formulated the theory of coupled thermoelasticity (named as C-D theory) to eliminate the paradox inherent in the classical uncoupled theory of thermoelasticity that the elastic changes have no effect on the temperature. But, the classical dynamical coupled theory of thermoelasticity still based on a parabolic heat equation, which predicts an infinite speed for the propagation of heat wave, contradicts the physical facts. Generalized theories of thermoelasticity have been developed that are free from this paradox. Lord and Shulman [2] (L-S theory) introduced the theory of generalized thermoelasticity based on a new law of heat conduction by incorporating a flux rate term and involved a hyperbolic type of heat transport equation (called the generalized thermoelasticity with one relaxation time). The L-S theory was extended by Dhaliwal and Sherief [3] to the case of anisotropic media. Uniqueness of the solution for the generalized thermoelasticity with one relaxation time under a variety of conditions was proved by Ignaczak [4] and Sherief and Dhaliwal [5] respectively. Generalized theory of thermoelasticity with two relaxation time parameters has also been proposed. Based on a generalized inequality of thermodynamics, Green and Lindsay [6] developed the theory of thermoelasticity with two relaxation time parameters (named as G-L theory). The G-L theory doesn't violate the Fourier's law of heat conduction when the body under consideration has a center of symmetry. In this theory, both the equations of motion and heat conduction are hyperbolic but the equation of motion is modified and differs from that of the classical dynamical coupled theory of thermoelasticity.

The axisymetric multilayered hollow cylinder problems have been discussed by some researchers in the uncoupled, coupled and generalized thermoelasticity in the recent years. Jane and Lee [7] considered the thermoelasticity of multilayered cylinders subjected to known temperatures at traction-free boundaries by using Laplace transform and the finite difference method. Kandil 
[8] studied the effect of steady-state temperature and pressure gradient on compound cylinders fitted together by shrink fit. Sherief and Anwar [9] discussed the problem of an infinitely long elastic circular cylinder whose inner and outer surfaces are subject to known temperature and traction free. Yang and Chen [10] discussed the transient response of one-dimensional quasi-static coupled thermoelasticity problems of an infinitely long annular cylinder composed of two different materials. Lee [11] solved the two-dimensional, quasi-static coupled, thermoelastic problem of finitely long hollow cylinder composed of two different materials with axial symmetry. Chen et al. $[12,13]$ discussed also the transient response of one-dimensional quasi static coupled and uncoupled thermoelasticity problems of multilayered hollow cylinder. Allam et al. [14] solved the problem of an infinite body with a circular cylindrical hole in a harmonic field in the context of the generalized theory of thermoelasticity. In a recent article, Zenkour et al. [15] presented the static bending response for a simply supported functionally graded rectangular plate subjected to a through-thethickness temperature field under the effect of various theories of generalized thermoelasticity with relaxation times.

In the present article, the analytical expressions for displacements, temperature and stresses of finitely long hollow cylinder composed of two different materials with axial symmetry are found in final forms. Numerical examples have been taken to discuss the effect of the relaxation times. Finally, the results have been illustrated graphically to find the differences between the different generalized theories of thermoelasticity.

\section{FORMULATION OF THE PROBLEM}

Through this area of research, we consider the following boundary value problem. We deal with a problem of finitely long hollow cylinder composed of three layered of two different materials with axial symmetry. The length of the multilayered hollow cylinder is $L$, and the inner and outer radii of the cylinder are denoted by $r_{i}$ and $r_{o}$, respectively (see Figure 1).

We assume that, the hollow cylinder is taken to be heated suddenly at the inner and outer surface under temperature $\phi_{i}$ and $\phi_{o}$, respectively. We take into account that the body forces are absent, and then the fundamental equations of the boundary value problem in the context of the different theories of generalized thermoelasticity, in the case of quasi-static, can be written as:

1) Equilibrium equations for the cylinder along $r$ and $z$ directions:

$$
\begin{gathered}
\sigma_{r}+r \frac{\partial \sigma_{r}}{\partial r}+r \frac{\partial \sigma_{r z}}{\partial z}-\sigma_{\theta}=0 \\
\sigma_{r z}+r \frac{\partial \sigma_{z}}{\partial z}+r \frac{\partial \sigma_{r z}}{\partial r}=0
\end{gathered}
$$

where $\sigma_{i j}$ are the components of stress tensor and $(r, \theta, z)$ are the cylindrical coordinate system.

2) General heat conduction equation in the context of generalized thermo-elasticity theories:

$$
\kappa_{r} \frac{\partial^{2} \bar{\Theta}}{\partial r^{2}}+\frac{\kappa_{\theta}}{r} \frac{\partial \bar{\Theta}}{\partial r}+\kappa_{z} \frac{\partial^{2} \bar{\Theta}}{\partial z^{2}}=\rho c_{v} \frac{\partial}{\partial t}\left(1+t_{2} \frac{\partial}{\partial t}\right) \bar{\Theta}+\Theta_{o}\left[\beta_{r} \frac{\partial}{\partial t}\left(1+t_{3} \frac{\partial}{\partial t}\right) \varepsilon_{r}+\beta_{\theta} \frac{\partial}{\partial t}\left(1+t_{3} \frac{\partial}{\partial t}\right) \varepsilon_{\theta}+\beta_{z} \frac{\partial}{\partial t}\left(1+t_{3} \frac{\partial}{\partial t}\right) \varepsilon_{z}\right],
$$

in which $\bar{\Theta}=\Theta-\Theta_{0}$ is the temperature and $\Theta_{0}$ is the reference temperature; $\varepsilon_{i}$ are the components of strain tensor; $t_{2}$ and $t_{3}$ are the second and third thermal relaxation times; $\kappa_{r}, \kappa_{\theta}$ and $\kappa_{z}$ are the thermal con- ductivity; $\rho$ is the density; and $c_{v}$ is the specific heat at constant deformation. The components of the thermoelastic tensor are given by

$$
\begin{gathered}
\beta_{r}=\frac{1}{\Delta}\left[E_{r}\left(1-v_{\theta z} v_{z \theta}\right) \alpha_{r}+E_{\theta}\left(v_{r \theta}+v_{\theta z} v_{r z}\right) \alpha_{\theta}+E_{z}\left(v_{r z}+v_{r \theta} v_{z \theta}\right) \alpha_{z}\right], \\
\beta_{\theta}=\frac{1}{\Delta}\left[E_{\theta}\left(v_{r \theta}+v_{\theta z} v_{r z}\right) \alpha_{r}+E_{\theta}\left(1-v_{r z} v_{z r}\right) \alpha_{\theta}+E_{z}\left(v_{\theta z}+v_{r \theta} v_{r z}\right) \alpha_{z}\right], \\
\beta_{z}=\frac{1}{\Delta}\left[E_{z}\left(v_{r z}+v_{r \theta} v_{\theta z}\right) \alpha_{r}+E_{z}\left(v_{\theta z}+v_{r \theta} v_{r z}\right) \alpha_{\theta}+E_{z}\left(1-v_{r \theta} v_{\theta r}\right) \alpha_{z}\right], \Delta=1-v_{r \theta} v_{\theta r}-v_{z \theta} v_{\theta z}-v_{z r} v_{r z}-2 v_{\theta r} v_{z \theta} v_{r z} .
\end{gathered}
$$

where $v_{r z}, v_{r \theta}$ and $v_{\theta z}$ are Poisson's ratios; $E_{r}, E_{\theta}$ and $E_{z}$ are Young's moduli; and $\alpha_{r}, \alpha_{\theta}$ and $\alpha_{z}$ are linear thermal expansion coefficients.

3) Duhamel-Neumann's relations for layer number $k$ :

$$
\begin{gathered}
\sigma_{r}=c_{11} \frac{\partial U_{r}}{\partial r}+c_{12} \frac{U_{r}}{r}+c_{13} \frac{\partial U_{z}}{\partial z}-\beta_{r}\left(1+t_{1} \frac{\partial}{\partial t}\right) \bar{\Theta}, \\
\sigma_{\theta}=c_{12} \frac{\partial U_{r}}{\partial r}+c_{22} \frac{U_{r}}{r}+c_{23} \frac{\partial U_{z}}{\partial z}-\beta_{\theta}\left(1+t_{1} \frac{\partial}{\partial t}\right) \bar{\Theta},
\end{gathered}
$$

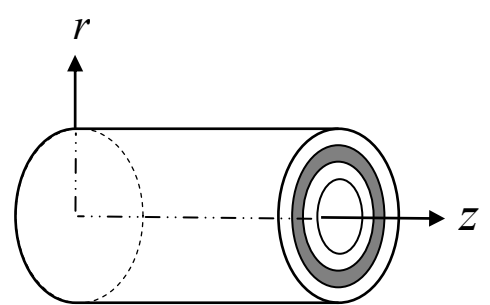

Figure 1. The three-layer hollow cylinder and its coordinate system. 


$$
\begin{gathered}
\sigma_{z}=c_{13} \frac{\partial U_{r}}{\partial r}+c_{23} \frac{U_{r}}{r}+c_{33} \frac{\partial U_{z}}{\partial z}-\beta_{z}\left(1+t_{1} \frac{\partial}{\partial t}\right) \bar{\Theta}, \\
\sigma_{r z}=c_{55}\left(\frac{\partial U_{z}}{\partial r}+\frac{\partial U_{r}}{\partial z}\right),
\end{gathered}
$$

where $t_{1}$ is the first thermal relaxation time and $U_{i}$ are the components of displacement vector. The elastic constants $c_{i j}$ are given by

$$
\begin{aligned}
& c_{11}=\frac{E_{r}\left(1-v_{\theta z} v_{z \theta}\right)}{\Delta}, \quad c_{22}=\frac{E_{\theta}\left(1-v_{r z} v_{z r}\right)}{\Delta}, \\
& c_{33}=\frac{E_{z}\left(1-v_{r \theta} v_{\theta r}\right)}{\Delta} \text {, } \\
& c_{12}=\frac{E_{\theta}\left(v_{r \theta}+v_{z \theta} v_{r z}\right)}{\Delta}=\frac{E_{r}\left(v_{\theta r}+v_{\theta z} v_{z r}\right)}{\Delta}=c_{21}, c_{55}=G_{r z} \text {, } \\
& c_{11} \frac{\partial^{2} U_{r}}{\partial r^{2}}+c_{11} \frac{1}{r} \frac{\partial U_{r}}{\partial r}-c_{22} \frac{U_{r}}{r^{2}}+c_{55} \frac{\partial^{2} U_{r}}{\partial z^{2}}+\left(c_{13}+c_{55}\right) \frac{\partial^{2} U_{z}}{\partial r \partial z} \\
& +\left(c_{13}-c_{23}\right) \frac{1}{r} \frac{\partial U_{z}}{\partial z}-\beta_{r} \frac{\partial}{\partial r}\left(1+t_{1} \frac{\partial}{\partial t}\right) \bar{\Theta}-\left(\beta_{r}-\beta_{\theta}\right) \frac{1}{r}\left(1+t_{1} \frac{\partial}{\partial t}\right) \bar{\Theta}=0, \\
& c_{55} \frac{\partial^{2} U_{z}}{\partial r^{2}}+\left(c_{13}+c_{55}\right) \frac{\partial^{2} U_{r}}{\partial r \partial z}+c_{55} \frac{1}{r} \frac{\partial U_{z}}{\partial r}+c_{33} \frac{\partial^{2} U_{z}}{\partial z^{2}} \\
& +\left(c_{23}+c_{55}\right) \frac{1}{r} \frac{\partial U_{r}}{\partial z}-\beta_{z} \frac{\partial}{\partial z}\left(1+t_{1} \frac{\partial}{\partial t}\right) \bar{\Theta}=0, \\
& {\left[\kappa_{r} \frac{\partial^{2}}{\partial r^{2}}+\frac{\kappa_{\theta}}{r} \frac{\partial}{\partial r}+\kappa_{z} \frac{\partial^{2}}{\partial z^{2}}\right] \bar{\Theta}=\rho c_{v} \frac{\partial}{\partial t}\left(1+t_{2} \frac{\partial}{\partial t}\right) \bar{\Theta}+\Theta_{o}\left[\beta_{r} \frac{\partial^{2}}{\partial r \partial t}\left(1+t_{3} \frac{\partial}{\partial t}\right) U_{r}\right.} \\
& \left.+\beta_{\theta} \frac{1}{r} \frac{\partial}{\partial t}\left(1+t_{3} \frac{\partial}{\partial t}\right) U_{r}+\beta_{z} \frac{\partial^{2}}{\partial z \partial t}\left(1+t_{3} \frac{\partial}{\partial t}\right) U_{z}\right]
\end{aligned}
$$

Eqs.10 and 11 represent the equations of equilibrium for the hollow cylinder along the $r$ and $z$ directions, respectively, while Eq.12 represents the coupled transient heat conduction equation for the $k$ th layer of the axisymmetric hollow cylinder. The boundary and interface conditions of the present composite hollow cylinder are given by:

\subsection{Boundary Conditions}

$$
\begin{aligned}
& U_{r}=U_{z}=0, \quad \bar{\Theta}=\eta_{1} \quad \text { at } \quad r=r_{i}, \\
& U_{r}=U_{z}=0, \quad \frac{\partial \bar{\Theta}}{\partial r}=0 \quad \text { at } r=r_{o}, \\
& \sigma_{r z}=U_{z}=0, \quad \bar{\Theta}=\eta_{2} \quad \text { at } \quad z=0, \\
& \sigma_{r z}=U_{z}=0, \quad \bar{\Theta}=\eta_{3} \quad \text { at } \quad z=L \text {. }
\end{aligned}
$$

\subsection{Interface Conditions}

$\left(U_{r}, U_{z}, \bar{\Theta}, \sigma_{r}, \sigma_{\theta}, \sigma_{z}, \sigma_{r z}\right)_{k}=\left(U_{r}, U_{z}, \bar{\Theta}, \sigma_{r}, \sigma_{\theta}, \sigma_{z}, \sigma_{r z}\right)_{k+1}$.

\subsection{Initial Conditions}

$$
\bar{\Theta}=\frac{1}{2} \Theta_{0} \text { at } t=0 .
$$

To solve the above equations, we introduce the following dimensionless quantities:

$$
\begin{gathered}
R=\frac{r}{r_{o}}, \quad Z=\frac{z}{L}, \quad \tau=\frac{t}{r_{o}^{2}}\left(\frac{\kappa_{r}}{\rho c_{v}}\right)_{1}, \quad t_{i}^{*}=\frac{t_{i}}{r_{o}^{2}}\left(\frac{\kappa_{r}}{\rho c_{v}}\right)_{1}, \\
i=1,2,3, \quad U_{r}^{*}=\frac{U_{r}}{r_{o}}\left(\frac{\beta_{r}}{\rho c_{v}}\right)_{1}, \quad U_{z}^{*}=\frac{U_{z}}{r_{o}}\left(\frac{\beta_{r}}{\rho c_{v}}\right)_{1}, \\
\sigma_{i j}^{*}=\frac{\sigma_{i j}}{\left(\beta_{r}\right)_{1}}, \quad R_{i}=\frac{r_{i}}{r_{o}}, \quad T^{*}=\frac{\Theta}{\Theta_{0}}, \quad \beta_{r}^{*}=\frac{\beta_{r}}{\left(\beta_{r}\right)_{1}}, \\
\kappa_{r}^{*}=\frac{\kappa_{r}}{\left(\kappa_{r}\right)_{1}}, \quad \frac{\beta_{\theta}}{\left(\beta_{r}\right)_{1}}, \quad \beta_{z}^{*}=\frac{\beta_{z}}{\left(\kappa_{r}\right)_{1}}, \quad c_{i j}^{*}=\frac{c_{i j}}{\left(c_{11}\right)_{1}}, \quad \kappa_{z}^{*}=\frac{\kappa_{z}}{\left(\kappa_{r}\right)_{1}}, \quad \rho c_{v}^{*}=\frac{\rho c_{v}}{\left(\rho c_{v}\right)_{1}}, \\
B_{r}=\beta_{r}\left(\frac{\beta_{r}}{\rho c_{v} c_{11}}\right)_{1} \Theta_{0}, \quad B_{\theta}=\beta_{\theta}\left(\frac{\beta_{r}}{\rho c_{v} c_{11}}\right)_{1} \Theta_{0},
\end{gathered}
$$




$$
\begin{aligned}
& B_{z}=\beta_{z}\left(\frac{\beta_{r}}{\rho c_{v} c_{11}}\right)_{1} \Theta_{0} . \\
& c_{11}^{*} \frac{\partial^{2} U_{r}^{*}}{\partial R^{2}}+c_{11}^{*} \frac{1}{R} \frac{\partial U_{r}^{*}}{\partial R}-c_{22}^{*} \frac{U_{r}^{*}}{R^{2}}+c_{55}^{*}\left(\frac{r_{o}}{L}\right)^{2} \frac{\partial^{2} U_{r}^{*}}{\partial Z^{2}}+\left(c_{13}^{*}+c_{55}^{*}\right) \frac{r_{o}}{L} \frac{\partial^{2} U_{z}^{*}}{\partial R \partial Z}+\left(c_{13}^{*}-c_{23}^{*}\right) \frac{r_{o}}{L} \frac{1}{R} \frac{\partial U_{z}^{*}}{\partial Z}-B_{r} \frac{\partial}{\partial R}\left(1+t_{1}^{*} \frac{\partial}{\partial \tau}\right) T^{*} \\
& -\left(B_{r}-B_{\theta}\right) \frac{1}{R}\left(1+t_{1}^{*} \frac{\partial}{\partial \tau}\right) T^{*}=0, \\
& c_{55}^{*} \frac{\partial^{2} U_{z}^{*}}{\partial R^{2}}+\left(c_{13}^{*}+c_{55}^{*}\right) \frac{r_{o}}{L} \frac{\partial^{2} U_{r}^{*}}{\partial R \partial Z}+c_{55}^{*} \frac{1}{R} \frac{\partial U_{z}^{*}}{\partial R}+c_{33}^{*}\left(\frac{r_{o}}{L}\right)^{2} \frac{\partial^{2} U_{z}^{*}}{\partial Z^{2}}+\left(c_{23}^{*}+c_{55}^{*}\right) \frac{r_{o}}{L} \frac{1}{R} \frac{\partial U_{r}^{*}}{\partial Z}-B_{z} \frac{r_{o}}{L} \frac{\partial}{\partial Z}\left(1+t_{1}^{*} \frac{\partial}{\partial \tau}\right) T^{*}=0, \\
& {\left[\kappa_{r}^{*} \frac{\partial^{2}}{\partial R^{2}}+\frac{\kappa_{\theta}^{*}}{R} \frac{\partial}{\partial R}+\kappa_{z}^{*}\left(\frac{r_{o}}{L}\right)^{2} \frac{\partial^{2}}{\partial Z^{2}}\right] T^{*}=\rho c_{v}^{*} \frac{\partial}{\partial \tau}\left(1+t_{2}^{*} \frac{\partial}{\partial \tau}\right) T^{*}} \\
& +\beta_{r}^{*} \frac{\partial^{2}}{\partial R \partial \tau}\left(1+t_{3}^{*} \frac{\partial}{\partial \tau}\right) U_{r}^{*}+\beta_{\theta}^{*} \frac{1}{R} \frac{\partial}{\partial \tau}\left(1+t_{3}^{*} \frac{\partial}{\partial \tau}\right) U_{r}^{*}+\beta_{z}^{*} \frac{r_{o}}{L} \frac{\partial^{2}}{\partial Z \partial \tau}\left(1+t_{3}^{*} \frac{\partial}{\partial \tau}\right) U_{z}^{*} .
\end{aligned}
$$

The dimensionless stresses are also given by

$$
\begin{array}{r}
\sigma_{r}^{*}=\bar{c}_{11} \frac{\partial U_{r}^{*}}{\partial R}+\bar{c}_{12} \frac{U_{r}^{*}}{R}+\bar{c}_{13} \frac{r_{o}}{L} \frac{\partial U_{z}^{*}}{\partial Z}-\beta_{r}^{*}\left(1+t_{1}^{*} \frac{\partial}{\partial \tau}\right) T^{*}, \\
\sigma_{\theta}^{*}=\bar{c}_{12} \frac{\partial U_{r}^{*}}{\partial R}+\bar{c}_{22} \frac{U_{r}^{*}}{R}+\bar{c}_{23} \frac{r_{o}}{L} \frac{\partial U_{z}^{*}}{\partial Z}-\beta_{\theta}^{*}\left(1+t_{1}^{*} \frac{\partial}{\partial \tau}\right) T^{*}, \\
\sigma_{z}^{*}=\bar{c}_{13} \frac{\partial U_{r}^{*}}{\partial R}+\bar{c}_{23} \frac{U_{r}^{*}}{R}+\bar{c}_{33} \frac{r_{o}}{L} \frac{\partial U_{z}^{*}}{\partial Z}-\beta_{z}^{*}\left(1+t_{1}^{*} \frac{\partial}{\partial \tau}\right) T^{*}, \\
\sigma_{r z}^{*}=\bar{c}_{55}\left(\frac{\partial U_{z}^{*}}{\partial R}+\frac{r_{o}}{L} \frac{\partial U_{r}^{*}}{\partial Z}\right), \\
T^{*}=\varphi(\tau) f_{1}(R) \cos (\pi Z), \\
U_{r}^{*}=u(\tau) f_{2}(R) \cos (\pi Z), \\
U_{z}^{*}=w(\tau) f_{2}(R) \sin (\pi Z),
\end{array}
$$

where

$$
\bar{c}_{i j}=\frac{c_{i j}}{\Theta_{0}}\left(\frac{\rho c_{v}}{\beta_{r}^{2}}\right)_{1} .
$$

The solution of Eqs.16-18 may be given by using the following substitutions of $T^{*}, U_{r}^{*}$ and $U_{z}^{*}$ that satisfy the boundary conditions given in Eq.13:

where the functions $f_{1}$ and $f_{2}$ are given, respectively, by:

$$
\begin{aligned}
& f_{1}=(R-1)^{2}-\left(R_{i}-1\right)^{2}+\Omega, \\
& f_{2}=\left(R-R_{i}\right)(R-1) .
\end{aligned}
$$

Using Eq.23 into Eqs.15-17, one can get

$$
\begin{aligned}
& A_{1}\left(1+t_{1}^{*} \frac{\mathrm{d}}{\mathrm{d} \tau}\right) \varphi+A_{2} u+A_{3} w=0, \quad A_{4}\left(1+t_{1}^{*} \frac{\mathrm{d}}{\mathrm{d} \tau}\right) \varphi+A_{5} u+A_{6} w=0, \\
& A_{7} \varphi=A_{8} \frac{\mathrm{d}}{\mathrm{d} \tau}\left(1+t_{3}^{*} \frac{\mathrm{d}}{\mathrm{d} \tau}\right) u+A_{9} \frac{\mathrm{d}}{\mathrm{d} \tau}\left(1+t_{3}^{*} \frac{\mathrm{d}}{\mathrm{d} \tau}\right) w+A_{10} \frac{\mathrm{d}}{\mathrm{d} \tau}\left(1+t_{2}^{*} \frac{\mathrm{d}}{\mathrm{d} \tau}\right) \varphi
\end{aligned}
$$

where

$$
\begin{gathered}
A_{1}=B_{2} \frac{f_{1}}{R}-B_{1}\left(\frac{1}{R}+\frac{\mathrm{d}}{\mathrm{d} R}\right) f_{1}, \quad A_{2}=c_{11}^{*}\left(\frac{1}{R} \frac{\mathrm{d}}{\mathrm{d} R}+\frac{\mathrm{d}^{2}}{\mathrm{~d} R^{2}}\right) f_{2}-c_{22}^{*} \frac{f_{2}}{R}-c_{55}^{*}\left(\frac{r_{o} \pi}{L}\right)^{2} f_{2}, \\
A_{3}=-\frac{r_{o} \pi}{L}\left[c_{13}^{*}\left(\frac{1}{R}+\frac{\mathrm{d}}{\mathrm{d} R}\right)-c_{23}^{*} \frac{1}{R}+\frac{\mathrm{d}}{\mathrm{d} R} c_{55}^{*}\right] f_{2}, \quad A_{4}=-\frac{r_{o} \pi}{L} B_{3} f_{1}, \\
A_{5}=\frac{r_{o} \pi}{L}\left[c_{55}^{*}\left(\frac{1}{R}+\frac{\mathrm{d}}{\mathrm{d} R}\right)+c_{23}^{*} \frac{1}{R}+\frac{\mathrm{d}}{\mathrm{d} R} c_{13}^{*}\right] f_{2}, \quad A_{6}=c_{55}^{*}\left(\frac{1}{R} \frac{\mathrm{d}}{\mathrm{d} R}+\frac{\mathrm{d}^{2}}{\mathrm{~d} R^{2}}\right) f_{2}-c_{33}^{*}\left(\frac{r_{o} \pi}{L}\right)^{2} f_{2},
\end{gathered}
$$




$$
A_{7}=\left(\kappa_{r}^{*} \frac{\mathrm{d}^{2}}{\mathrm{~d} R^{2}}+\kappa_{\theta}^{*} \frac{1}{R} \frac{\mathrm{d}}{\mathrm{d} R}-\kappa_{z}^{*}\left(\frac{r_{o} \pi}{L}\right)^{2}\right) f_{1}, \quad A_{8}=\left(\beta_{r}^{*} \frac{\mathrm{d}}{\mathrm{d} R}+\beta_{\theta}^{*} \frac{1}{R}\right) f_{2}, \quad A_{9}=-\frac{r_{o} \pi}{L} \beta_{z}^{*} f_{2}, \quad A_{10}=\rho c_{v}^{*} f_{1} .
$$

The solution of the above system of first-order differential equations may be easily given for all theories. The corresponding solutions for C-D, L-S and G-L theories are also obtained from the general one.

\section{NUMERICAL EXAMPLES}

In order to illustrate the results graphically, the geometry and thermoelastic constants for the two materials of the hollow cylinder are given in Table 1 . The cylinder is composed of three layers of two distinct materials with the same thickness of each layer. Layers 1 and 3 have properties of the same material. So, the two interfaces are given at $R=0.5$ and $R=0.75$, respectively. The various non-dimensional parameters used are:

$$
\begin{gathered}
u_{1}=10^{2} U_{r}^{*}=\frac{U_{r}}{r_{o}}\left(\frac{\beta_{r}}{\rho c_{v}}\right)_{1}, \quad u_{3}=U_{z}^{*}=\frac{U_{z}}{r_{o}}\left(\frac{\beta_{r}}{\rho c_{v}}\right)_{1}, \\
T=T^{*}=\frac{\bar{\Theta}}{\Theta_{0}}, \sigma_{1}=\sigma_{r}^{*}=\frac{\sigma_{r}}{\left(\beta_{r}\right)_{1}}, \quad \sigma_{2}=\sigma_{\theta}^{*}=\frac{\sigma_{\theta}}{\left(\beta_{r}\right)_{1}}, \\
\sigma_{3}=\sigma_{z}^{*}=\frac{\sigma_{z}}{\left(\beta_{r}\right)_{1}}, \quad \sigma_{5}=\sigma_{r z}^{*}=\frac{\sigma_{r z}}{\left(\beta_{r}\right)_{1}} .
\end{gathered}
$$

The numerical results are plotted in Figures 2-17. The values of $\Theta_{0}$ and $\Omega$ are taken to have the same value as $\Theta_{0}=\Omega=5$. The values of $\phi_{i}$ and $\phi_{o}$ and $\eta_{1}, \eta_{2}$, and $\eta_{3}$ are given, respectively, in terms of $\Theta_{0}$ and $\Omega$. The ratio of the outer radius of the cylinder to its length is given by $r_{o} / L=0.2$. In addition, the $*$ is dropped, for simplicity, from the dimensionless relaxation times.

Figure 2 illustrates the variation of dimensionless temperature $T$ through axial parameter $Z$, for value of the dimensionless time namely $\tau=8$ and at the second interface of the dimensionless radial direction $(R=0.75)$. The computations were carried out for C-D, L-S and G-L theories of thermoelasticity. Figure 3 shows the variation of dimensionless radial stress $\sigma_{1}$ through the axial parameter $Z$. The values of dimensionless time and radial direction are chosen to be 8 and 0.75 , respectively. The results were calculated for L-S and G-L theories.

In what follows, we restrict our attention to the results of L-S theory. Figures 4,6,8,10,12,14 and 16 illustrate, respectively, the variation of dimensionless radial and axial displacements, $u_{1}$ and $u_{3}$; the dimensionless temperature $T$; and the dimensionless stresses $\sigma_{1}$, $\sigma_{2}, \sigma_{3}$, and $\sigma_{5}$ through the radial direction of the multilayered hollow cylinder for different values of the dimensionless time $\tau=5,7$, and 9 with the relaxation time $t_{3}=t_{2}=20$. Similar results are plotted in Figures

\begin{tabular}{|c|c|c|}
\hline & Material 1 & Material 2 \\
\hline$E_{r}\left(\frac{N}{m^{2}}\right)$ & $50 \times 10^{6}$ & $58 \times 10^{6}$ \\
\hline$E_{\theta}\left(\frac{N}{m^{2}}\right)$ & $15 \times 10^{6}$ & $22 \times 10^{6}$ \\
\hline$E_{z}\left(\frac{N}{m^{2}}\right)$ & $15 \times 10^{6}$ & $18 \times 10^{6}$ \\
\hline$G_{r z}\left(\frac{N}{m^{2}}\right)$ & $15 \times 10^{5}$ & $20 \times 10^{6}$ \\
\hline$\kappa_{r}\left(\frac{\text { watt }}{\mathrm{m} \cdot \mathrm{K}}\right)$ & 18 & 22 \\
\hline$\kappa_{\theta}\left(\frac{\text { watt }}{\mathrm{m} \cdot \mathrm{K}}\right)$ & 12 & 15 \\
\hline$\kappa_{z}\left(\frac{\text { watt }}{\mathrm{m} \cdot \mathrm{K}}\right)$ & 15 & 20 \\
\hline$v_{r \theta}=v_{\theta r}$ & 0.2 & 0.2 \\
\hline$v_{r z}=v_{z r}$ & 0.1 & 0.1 \\
\hline$v_{z \theta}=v_{\theta z}$ & 0.15 & 0.15 \\
\hline$\alpha_{\theta}=\alpha_{z}\left(\frac{1}{\mathrm{~K}}\right.$ & $3 \times 10^{-6}$ & $3 \times 10^{-6}$ \\
\hline$\alpha_{r}\left(\frac{1}{\mathrm{~K}}\right)$ & $4 \times 10^{-6}$ & $4 \times 10^{-6}$ \\
\hline$\rho\left(\frac{\mathrm{kg}}{\mathrm{m}^{3}}\right)$ & 0.095 & 0.095 \\
\hline$c_{v}\left(\frac{\mathrm{kj}}{\operatorname{kg} K}\right)$ & 0.31 & 0.31 \\
\hline
\end{tabular}
$\mathbf{5 , 7 , 9 , 1 1 , 1 3 , 1 5}$, and $\mathbf{1 7}$ through the radial direction of the
Table 1. The geometry and material constants of a finitely long hollow cylinder.

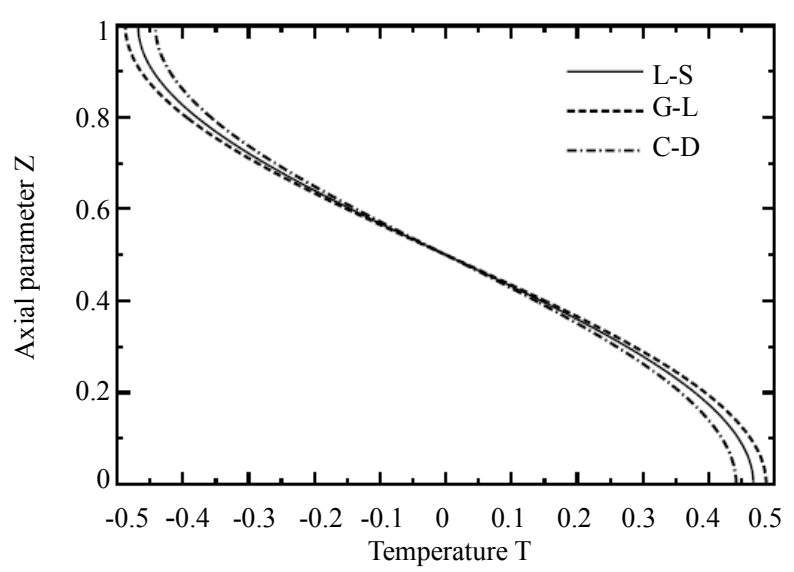

Figure 2. Variation of dimensionless temperature $T$ through the axial direction of the hollow cylinder for various thermoelasticity theories ( $\tau=8 ; R=0.75)$. 


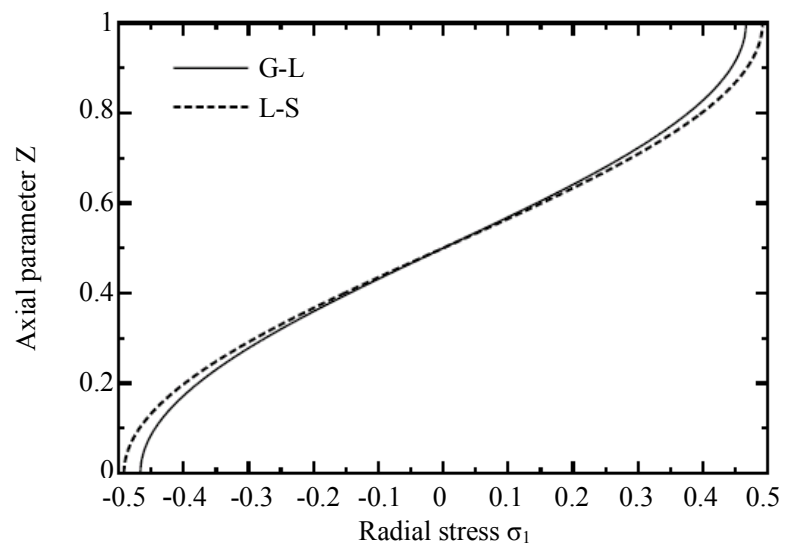

Figure 3. Variation of dimensionless radial stress $\sigma_{1}$ through the axial direction of the hollow cylinder for L-S and G-L theories ( $\tau=8 ; R=0.75)$.

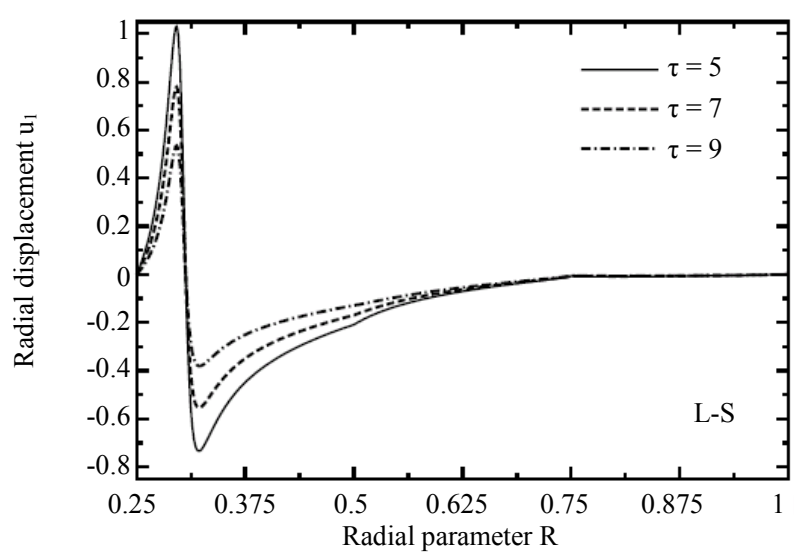

Figure 4. Variation of dimensionless radial displacement $u_{1}$ through the radial direction of the hollow cylinder for different values of the time parameter $\tau \quad\left(t_{2}=t_{3}=20\right)$.

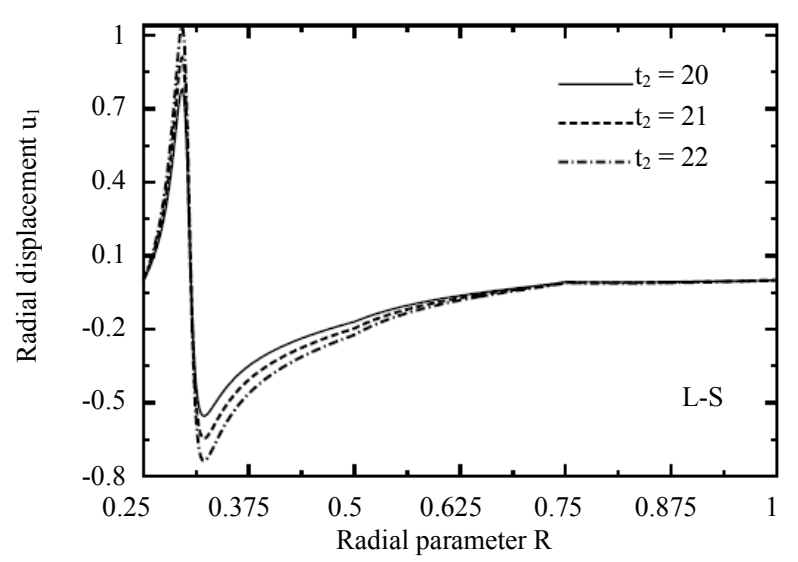

Figure 5. Variation of dimensionless radial displacement $u_{1}$ through the radial direction of the hollow cylinder for different values of the relaxation time $\left(t_{2}=t_{3}, \tau=7\right)$.

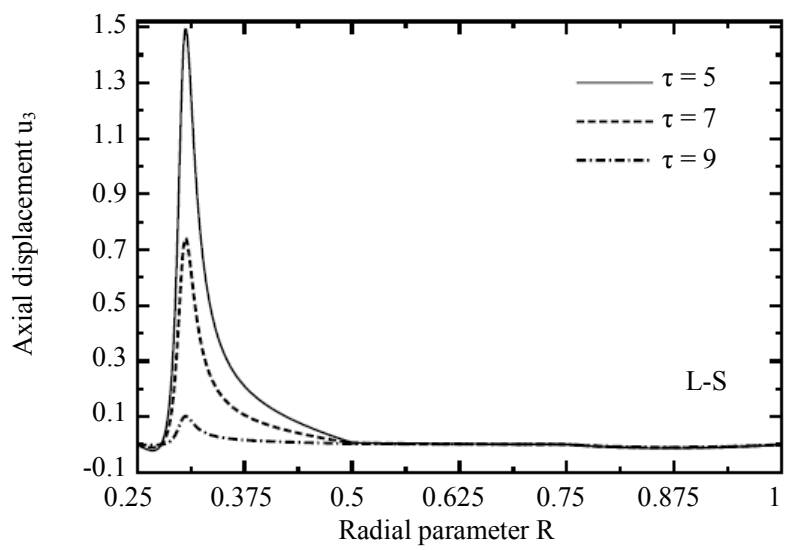

Figure 6. Variation of dimensionless axial displacement $u_{3}$ through the radial direction of the hollow cylinder for different values of the time parameter $\tau \quad\left(t_{2}=t_{3}=20\right)$.

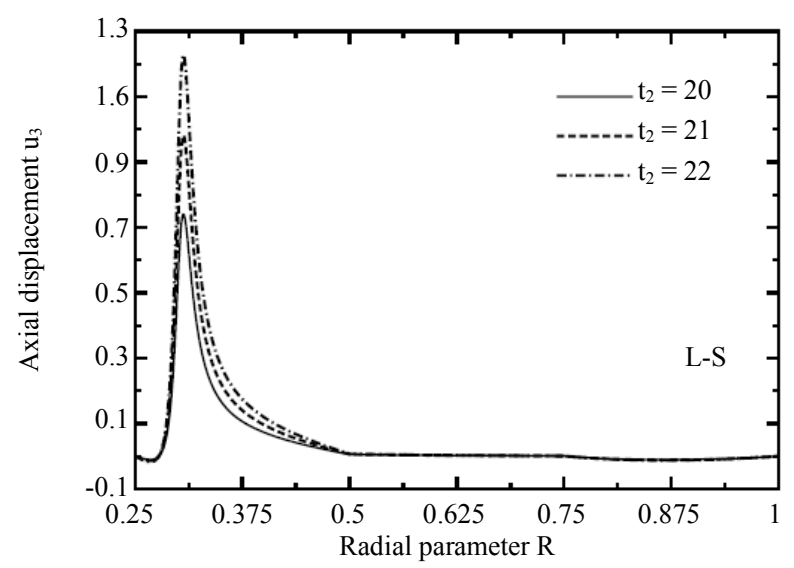

Figure 7. Variation of dimensionless axial displacement $u_{3}$ through the radial direction of the hollow cylinder for different values of the relaxation time $\left(t_{2}=t_{3}, \tau=7\right)$.

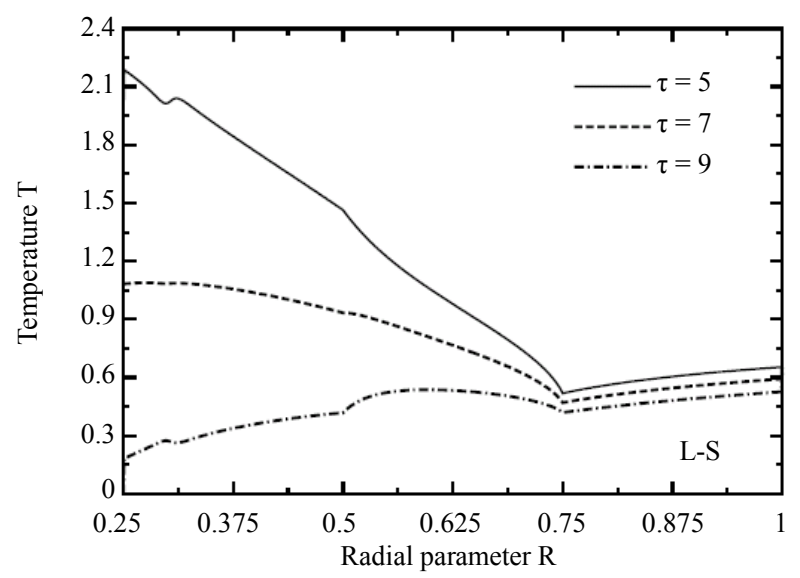

Figure 8. Variation of dimensionless temperature $T$ through the radial direction of the hollow cylinder for different values of the time parameter $\tau\left(t_{2}=t_{3}=20\right)$. 


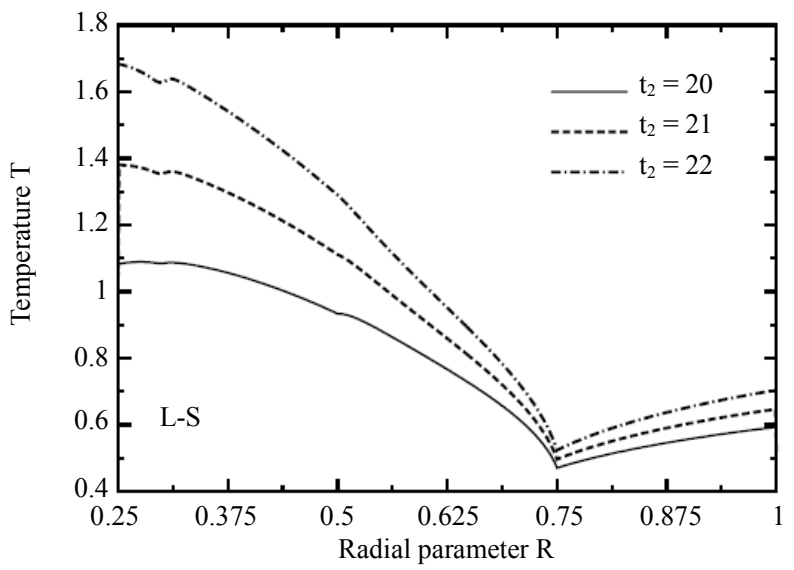

Figure 9. Variation of dimensionless temperature $T$ through the radial direction of the hollow cylinder for different values of the relaxation time $\left(t_{2}=t_{3}, \tau=7\right)$.

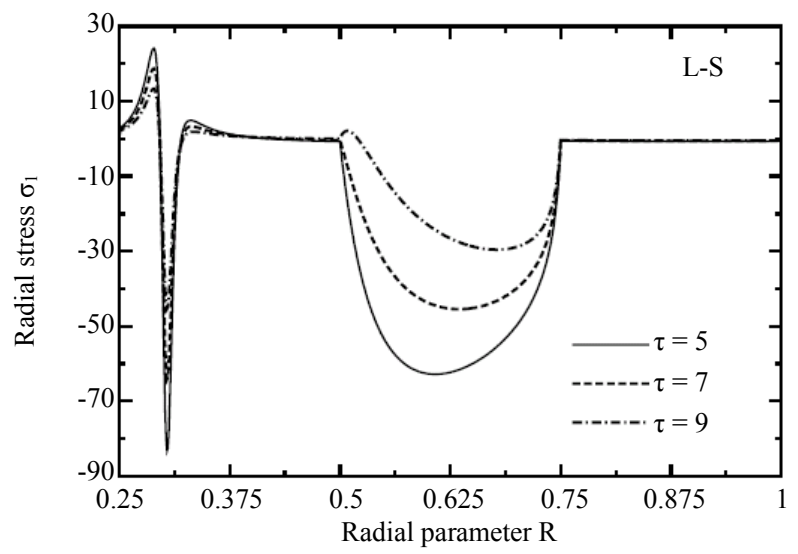

Figure 10. Variation of dimensionless radial stress $\sigma_{1}$ through the radial direction of the hollow cylinder for different values of the time parameter $\tau \quad\left(t_{2}=t_{3}=20\right)$.

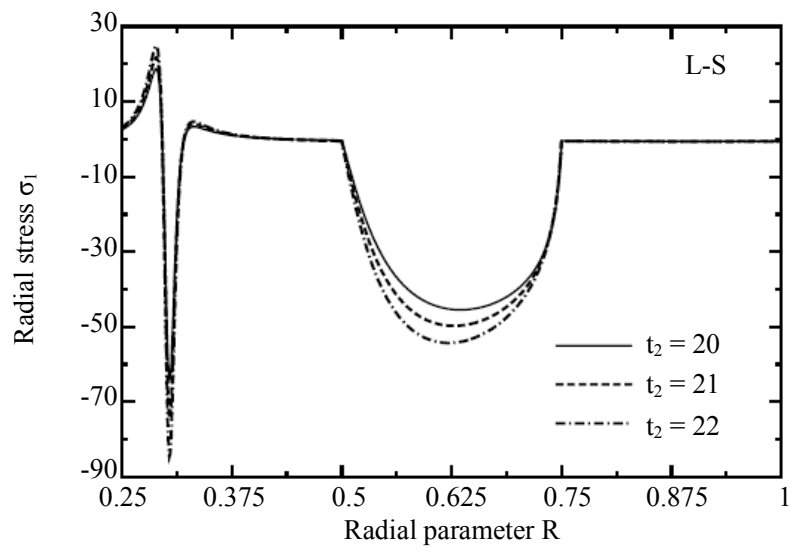

Figure 11. Variation of dimensionless radial stress $\sigma_{1}$ through the radial direction of the hollow cylinder for different values of the relaxation time $\left(t_{2}=t_{3}, \tau=7\right)$.

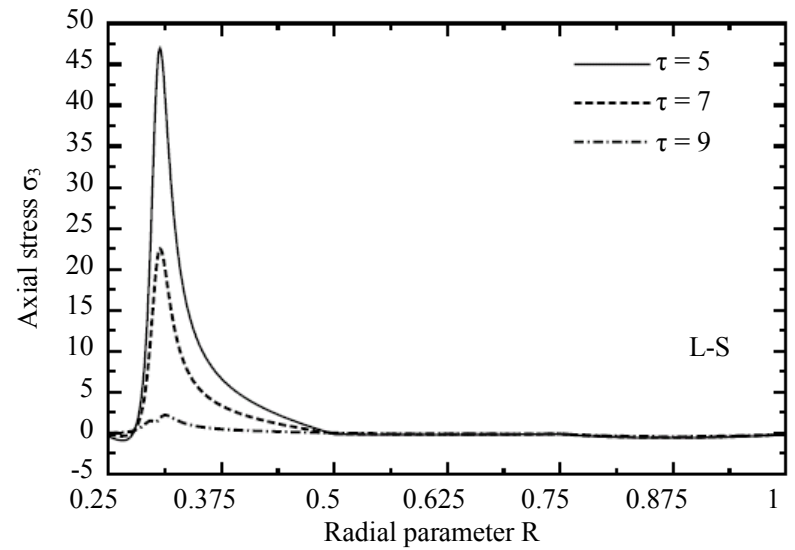

Figure 12. Variation of dimensionless axial stress $\sigma_{3}$ through the radial direction of the hollow cylinder for different values of the time parameter $\tau \quad\left(t_{2}=t_{3}=20\right)$.

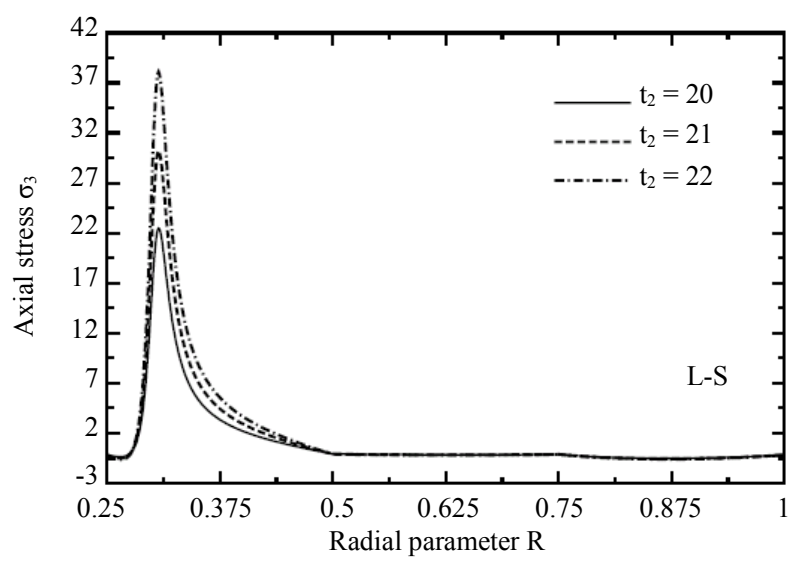

Figure 13. Variation of dimensionless axial stress $\sigma_{3}$ through the radial direction of the hollow cylinder for different values of the relaxation time $\left(t_{2}=t_{3}, \tau=7\right)$.

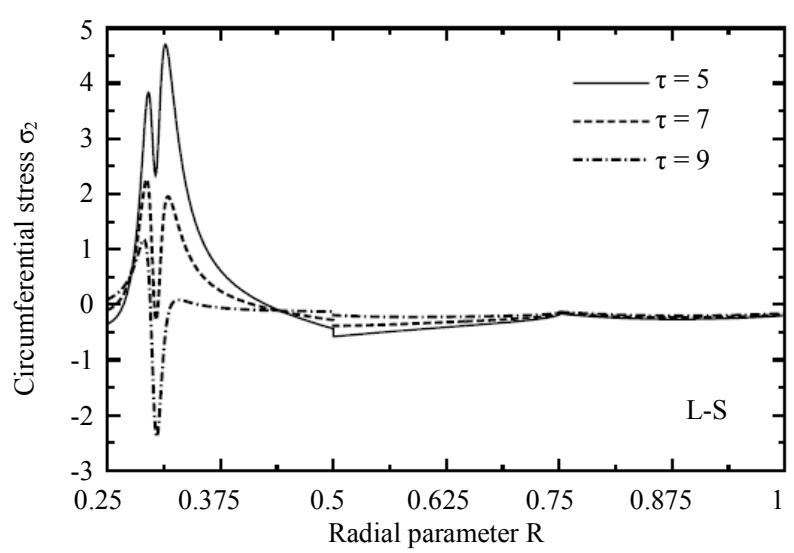

Figure 14. Variation of dimensionless circumferential stress $\sigma_{2}$ through the radial direction of the hollow cylinder for different values of the time parameter $\tau \quad\left(t_{2}=t_{3}=20\right)$. 


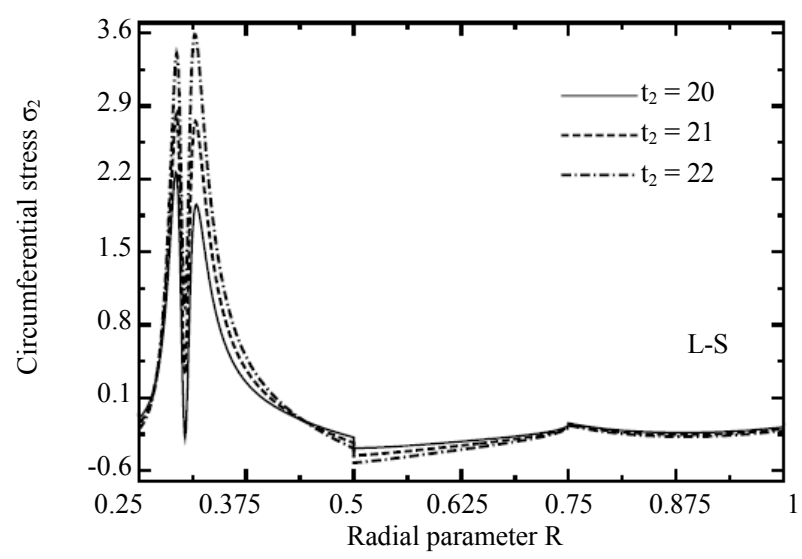

Figure 15. Variation of dimensionless circumferential stress $\sigma_{2}$ through the radial direction of the hollow cylinder for different values of the relaxation time $\left(t_{2}=t_{3}, \tau=7\right)$.

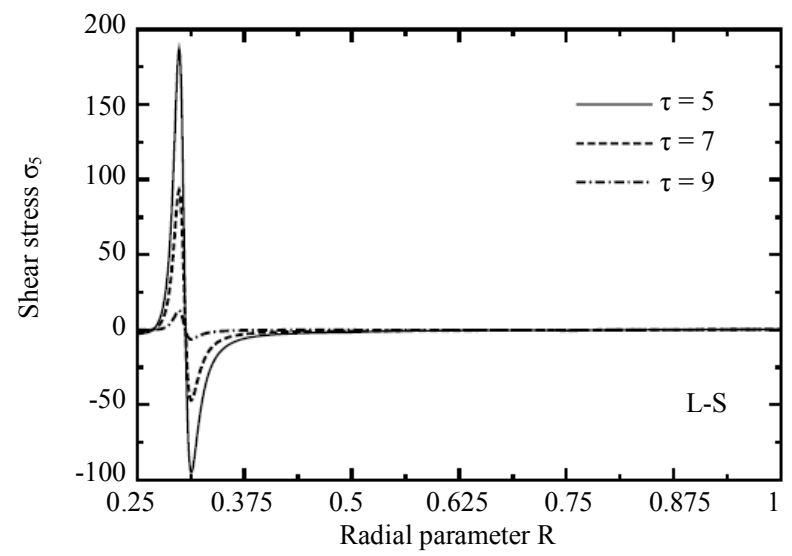

Figure 16. Variation of dimensionless shear stress $\sigma_{5}$ through the radial direction of the hollow cylinder for different values of the time parameter $\tau \quad\left(t_{2}=t_{3}=20\right)$.

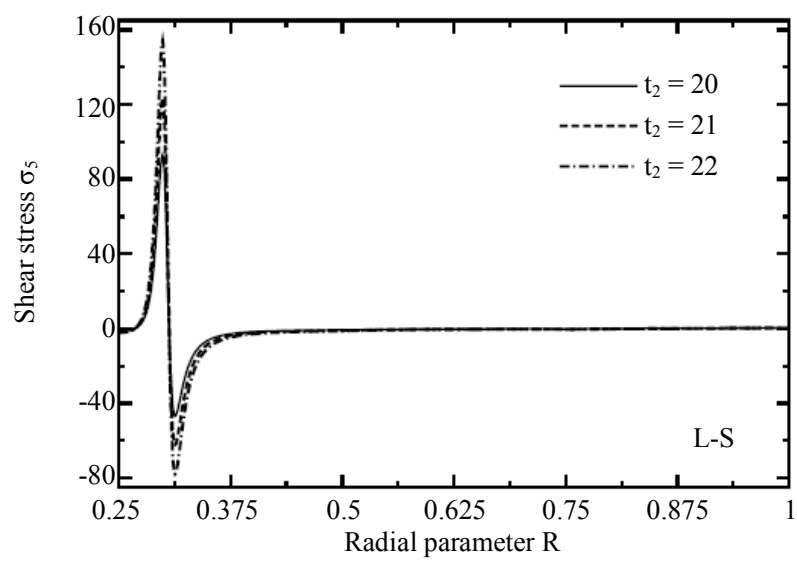

Figure 17. Variation of dimensionless shear stress $\sigma_{5}$ through the radial direction of the hollow cylinder for different values of the relaxation time $\left(t_{2}=t_{3}, \tau=7\right)$. multilayered hollow cylinder for different values of the relaxation time $\left(t_{3}=t_{2}=20,21,22\right)$ when the dimensionless time $\tau=7$.

\section{CONCLUSIONS}

The conclusion of the above results may be given as:

1) Figure 2 illustrates that the dimensionless temperature is slightly changed and the differences between $\mathrm{C}-\mathrm{D}, \mathrm{L}-\mathrm{S}$, and G-L are very small (tiny). The coupled theory (C-D) may give results with small relative error compared with those given by Lord and Shulman's (L-S) and Green and Lindsay's (G-L) theories. However, the results of L-S and G-L are much closed to each other (see Figure 3).

2) The plots of results given by Lord and Shulman's theory show that the effect of the dimensionless time is slightly clear in the first layer, but in the second and third layers the effect is not declared. This happened for dimensionless radial and axial displacements (see Figures 4-7), and axial, circumferential, and shear stresses (see Figures 12-17).

3) However, for dimensionless temperature and radial stress, the effects of dimensionless time is very clear in the first layer and start to decrease with the increase of radial direction in the second and third layer (see Figures 8-11).

4) The effect of the relaxation time of Lord and Shulman's theory in all physical waves (displacements, temperature and stresses) is clear in the first layer, but is less considerable in the second and third layers. This revealed that the effect of the relaxation time has no effect when the dimensionless radius is increasing.

\section{ACKNOWLEDGEMENT}

This paper is fully supported by the Deanship of Scientific Research at King AbdulAziz University, Grant No. 181/428.

\section{REFERENCES}

[1] Biot, M. (1956) Thermoelasticity and irreversible thermo-dynamics. Journal of Applied Physics, 27, 240-253.

[2] Lord, H.W. and Shulman, Y.A. (1967) A generalized dynamical theory of thermoelasticity. Jornal of Mechanics and Physics of Solids, 15, 299-309.

[3] Dhaliwal, R.S. and Sherief, H.H. (1980) Generalized thermoelasticity for anisotropic media. Quarterly of Applied Mathematics, 38, 1-8.

[4] Ignaczak, J. (1979) Uniqueness in generalized thermoelasticity. Journal of Thermal Stresses, 2, 171-175.

[5] Sherief, H.H. and Dhaliwal, R. (1980) A uniqueness theorem and a variational principle for generalized thermoelasticity. Journal of Thermal Stresses, 3, 223-230. 
[6] Green, A. and Lindsay, K. (1972) Thermoelasticity. Journal of Elasticity, 2, 1-7.

[7] Jane, K.C. and Lee, Z.Y. (1999) Thermoelasticity of multilayered cylinder. Journal of Thermal Stresses, 22, 57-74.

[8] Kandil, A. (1975) Investigation of stress analysis in compound cylinders under high pressure and temperature. M.Sc. Thesis, CIT Helwan.

[9] Sherief, H.H. and Anwar, M.N. (1988) A problem in generalized thermoelasticity for an infinitely long annular cylinder. International Journal of Engineering Science, 26, 301-306.

[10] Yan, Y.C. and Chen, C.K. (1986) Thermoelastic transient response of an infinitely long annular cylinder composed of two different materials. International Journal of Engineering Science, 24, 569-581.

[11] Lee, Z.-Y. (2006) Generalized coupled transient response of 3-D multilayered hollow cylinder. International Com- munications in Heat and Mass Transfer, 33, 1002-1012.

[12] Chen, C.K., Hung, C.I. and Lee, Z.Y. (2001) Transient thermal stresses analysis of multilayered hollow cylinder. Acta Mechanica, 151, 75-88.

[13] Chen, C.K., Hung, C.I. and Lee, Z.Y. (2001) Thermoelastic transient response of multilayered hollow cylinder with initial interface pressure. Journal of Thermal Stresses, 24, 987-1006.

[14] Allam, M.N., Elsibai, K.A. and Abouelregal, A.E. (2002) Thermal stresses in a harmonic field for an infinite body with a circular cylindrical hole without energy dissipation. Journal of Thermal Stresses, 25, 57-67.

[15] Zenkour, A.M., Mashat, D.S. and Elsibai, K.A. (2009) Bending analysis of functionally graded plates in the context of different theories of thermoelasticity. Mathematical Problems in Engineering, 2009, Article ID 962351, $1-15$. 